Revue internationale P.M.E.

Économie et gestion de la petite et moyenne entreprise

\title{
La succession dans les entreprises familiales dirigées par les femmes : une problématique en quête de chercheurs
}

\section{Louise Cadieux, Jean Lorrain et Pierre Hugron}

Volume 15, numéro 1, 2002

URI : https://id.erudit.org/iderudit/1008803ar

DOI : https://doi.org/10.7202/1008803ar

Aller au sommaire du numéro

Éditeur(s)

Presses de l’Université du Québec

ISSN

0776-5436 (imprimé)

1918-9699 (numérique)

Découvrir la revue

Citer ce document

Cadieux, L., Lorrain, J. \& Hugron, P. (2002). La succession dans les entreprises familiales dirigées par les femmes : une problématique en quête de chercheurs. Revue internationale P.M.E., 15(1), 115-130.

https://doi.org/10.7202/1008803ar
Résumé de l'article

La succession est l'un des volets ayant le plus été étudié en contexte d'entreprises familiales. Pourtant, bien que l'on évalue à plus de $33 \%$ la part des entreprises appartenant à des femmes, à notre connaissance, aucune étude ne s'est encore intéressée à la problématique dans leur cas. En conséquence, l'objectif de cet article est d'expliquer comment deux champs de recherche jusqu'ici séparés peuvent être complémentaires dans la manière d'aborder la problématique de la succession en général. Il s'agit donc d'un exercice de réflexion qui, somme toute, soulève un débat d'actualité sur l'état de nos connaissances sur les femmes entrepreneures en contexte d'entreprises familiales et qui se verront bientôt confrontées à une problématique de taille, celle de la succession. 


\title{
Note de recherche
}

\section{La succession dans les entreprises familiales dirigées par les femmes: une problématique en quête de chercheurs}

\author{
Louise CADIEUX \\ Jean LORRAIN \\ Université du Québec à Trois-Rivières \\ Pierre HUGRON
}

École des Hautes Études commerciales

\author{
MOTS CLÉS
}

Femmes entrepreneures - Succession - Entreprises familiales PME - Modes de gestion - Perspectives

\begin{abstract}
LES AUTEURS
LouISE CADIEUX est détentrice d'une maîtrise en gestion des PME et leur environnement. Elle est présentement chargée de cours en comportement organisationnel et en management alors qu'elle poursuit sa formation doctorale - DBA en administration à l'Université du Québec à Trois-Rivières. Elle s'intéresse plus précisément à l'étude des entreprises familiales et au processus de la succession. Adresse : Université du Québec à Trois-Rivières, Département des sciences de la gestion et de l'économie, 3351, boul. des Forges, Trois-Rivières, Québec, G9A 5H7. Courriel : <louise_cadieux@uqtr.ca>.

JEAN LORRAIN est détenteur d'un doctorat en psychologie industrielle, il est depuis plus de vingt-cinq ans professeur en psychologie des organisations à l'Université du Québec à TroisRivières. Reconnu mondialement pour ses travaux sur le profil psychologique et les compétences des entrepreneurs, il est à ce jour l'une des références les plus reconnues par le milieu universitaire et l'une des ressources-conseils les plus utilisées par les gens d'affaires. Adresse : Université du Québec à Trois-Rivières, Département des sciences de la gestion et de l'économie, 3351, boul. des Forges, Trois-Rivières, Québec, G9A 5H7. Courriel : <jean_lorrain@uqtr.ca>.

PIERRE HUGRon est professeur honoraire de l'École des Hautes Études commerciales, affiliée à l'Université de Montréal, professeur en management et en entrepreneurship et fondateur du Groupe de recherche sur les entreprises familiales (GREF). Il a enseigné et donné de nombreuses conférences en Amérique, en Europe, en Asie (Chine et Japon) et en Afrique. Adresse : École des Hautes Études commerciales, 2640, chemin du Lac-Supérieur, Lac-Supérieur, Québec, J0T 1P0.
\end{abstract}




\title{
RÉSUMÉ
}

La succession est l'un des volets ayant le plus été étudié en contexte d'entreprises familiales. Pourtant, bien que l'on évalue à plus de $33 \%$ la part des entreprises appartenant à des femmes, à notre connaissance, aucune étude ne s'est encore intéressée à la problématique dans leur cas. En conséquence, l'objectif de cet article est d'expliquer comment deux champs de recherche jusqu'ici séparés peuvent être complémentaires dans la manière d'aborder la problématique de la succession en général. Il s'agit donc d'un exercice de réflexion qui, somme toute, soulève un débat d'actualité sur l'état de nos connaissances sur les femmes entrepreneures en contexte d'entreprises familiales et qui se verront bientôt confrontées à une problématique de taille, celle de la succession.

\begin{abstract}
Succession is one of the most studied aspects of family businesses. However, while it has been estimated that more than $33 \%$ of such organizations are owned by women, to our knowledge no studies have yet to focus on succession in them. The objective of the present paper is therefore to show how two fields of research that have until now been separated can come together to provide insight into how to approach the problem of succession in general. It is intended to provide food for thought on a subject that is currently under discussion, i.e. what we know today about women who manage family-owned businesses and who will soon be confronted with a sizeable problem, that of succession.
\end{abstract}

\section{RESUMEN}

La sucesión es uno de los aspectos que más se ha estudiado en el contexto de empresas familiares. Sin embargo, aunque se ha evaluado en más del $33 \%$ de las empresas pertenecientes a mujeres, a nuestro conocimiento, nadie se interesa todavía en la problemática del caso. En consecuencia, el objetivo de este artículo es explicar cómo dos campos de investigación hasta ahora separados pueden ser complementarios en la forma de abordar la problemática de la sucesión en general. Se trata entonces de un ejercicio de reflexión que, en suma, propicia un debate de actualidad acerca del estado de nuestros conocimientos sobre las mujeres empresarias en el contexto de empresas familiares y que se verán muy pronto confrontradas a una problemática de gran dimensión, la de la sucesión.

\section{ZUSAMMENFASSUNG}

Die Nachfolgeregelung zählt zu den am meisten untersuchten Gebieten im Zusammenhang mit den Familienunternehmen. Obwohl mehr als $33 \%$ der Unternehmen Frauen gehören, hat nach unserer Kenntnis, keine Studie sich bis jetzt dieser Problematik gewidmet. Daraus erfolgte das Ziel dieses Artikels, nämlich zu klären, wie zwei sich bis jetzt getrennte Untersuchungsgebiete ergänzen können, um das Nachfolgeproblem allgemein zu erörtern.

Es handelt sich also um eine Überlegungsübung, die eine aktuelle Auseinandersetzung unterstreicht, und zwar über unsere Kenntnisse von Unternehmerfrauen in Familienunternehmen, welche sich oft mit der Nachfolgeregelung konfrontiert sehen.

Revue internationale P.M.E., vol. 15, nº 1, 2002 


\section{Introduction}

L'entrepreneuriat féminin fait depuis plus de vingt-cinq ans l'objet d'une certaine attention de la part de la communauté scientifique en entrepreneuriat, et ce, surtout, depuis la prise de conscience par les chercheurs du nombre de PME contrôlées et dirigées par des femmes et de l'accentuation de ce phénomène au cours des années. Par exemple, en 1997, au Québec et au Canada, les femmes représentaient près du tiers de l'ensemble des entrepreneurs comparativement au quart dix ans plus tôt (Ratté, 1999). En 1996, aux États-Unis, on estimait que les entreprises appartenant à des femmes généraient des revenus équivalant à 3,6 trillions de dollars et qu'elles procuraient de l'emploi à plus de 27,5 millions de personnes (Brush et Hisrich, 2000).

Selon Baker, Aldrich et Liou (1997), entre les années 1980 et 1990, le pourcentage d'articles publiés dans les revues scientifiques et professionnelles a diminué de manière significative. Ainsi, malgré l'importance grandissante de la place des femmes entrepreneures dans l'économie, de moins en moins de publications scientifiques leur sont consacrées. Selon Stevenson (1990), ce quasi-désintéressement de la part de la communauté scientifique en entrepreneuriat s'expliquerait en grande partie par le fait que la recherche soit majoritairement entre les mains de chercheurs masculins qui, par voie de conséquence, démontrent peu d'intérêt pour des problématiques féminines. En somme, pour l'auteure, l'entrepreneuriat est un phénomène de «gars pour des gars » où l'entrepreneuriat féminin n'apparaît que comme un champ d'étude mineur. Du reste, nous ne pouvons passer sous silence le fait que les connaissances accumulées à ce jour sur l'entrepreneuriat féminin soient, en grande partie, le fruit de chercheurs anglo-saxons, en particulier nord-américains et que très peu de chercheurs du monde francophone se sont penchés sur les problématiques propres aux femmes entrepreneures.

\section{Les paradigmes de recherches en entrepreneuriat féminin}

Historiquement, les premiers travaux sur les femmes entrepreneures s'inscrivent dans un paradigme normatif. D'entrée de jeu, tenant pour acquis que l'entrepreneuriat dépend davantage de l'expérience que du sexe ou de la personne, les femmes entrepreneures sont systématiquement comparées à leurs homologues masculins. La base comparative s'appuie sur des connaissances accumulées provenant de résultats de recherches majoritairement faites par sondage et ayant des échantillons composés exclusivement d'hommes (Stevenson, 1990). Les principales rubriques étudiées sont les caractéristiques personnelles et psychologiques des femmes entrepreneures, le profil et l'évolution de leurs entreprises, leur style de gestion et la particularité des problèmes qu'elles rencontrent.

Revue internationale P.M.E., vol. 15, nº 1, 2002 
Les tenants de l'école féministe critiquent vivement les résultats de toutes ces recherches qui, à leur avis, contribuent à l'édification d'un stéréotype entrepreneurial et à la négation des particularités intrinsèques de l'entrepreneuriat féminin à la faveur d'un impérialisme sexuel. Conséquemment, l'entrepreneuriat féminin étant un phénomène intrinsèquement différent de l'entrepreneuriat masculin, ceux-ci rejettent les travaux basés sur le modèle masculin optant plutôt pour des recherches faites exclusivement auprès des femmes entrepreneures, cela en préconisant des approches qualitatives en profondeur.

D'autres optent pour une position intermédiaire où, malgré tout, l'entrepreneuriat féminin demeure un phénomène particulier et différencié de l'entrepreneuriat masculin. L'approche intégrée, loin de rejeter les résultats des recherches normatives et moins radicales que les féministes, s'appuie sur des travaux réalisés dans les domaines de la sociologie et de la psychologie de la femme, d'une part, et, d'autre part, sur une réinterprétation des résultats de recherche provenant de l'école normative, en particulier sur les différences observées entre les hommes et les femmes entrepreneurs au regard des objectifs poursuivis en affaires et du style de management. Selon cette perspective soutenue par Brush (1992), par nature, «les femmes voient le monde des affaires comme un système d'interrelations plutôt qu'une structure divisée en deux entités distinctes composées d'une sphère économique et d'une sphère sociale qui ne se rejoignent pas. Les affaires sont, dans une perspective féminine, intégrées à l'ensemble de toutes leurs activités ${ }^{1} \gg$. Donc, contrairement à leurs homologues masculins, les femmes entrepreneures concevraient leurs activités d'affaires et leurs entreprises comme des réseaux coopératifs de relations plutôt que comme un centre de profits seulement. Et, comme nous le verrons dans les prochains paragraphes, parce qu'elle s'appuie sur des preuves empiriques pouvant expliquer la réalité particulière des femmes entrepreneures, cette école de pensée est, en ce qui nous concerne, celle qui fournit les meilleurs fondements théoriques.

\section{Les femmes entrepreneures : un monde à part}

L'une des premières interrogations des chercheurs intéressés au phénomène de l'entrepreneuriat féminin consistait à savoir comment les femmes se comportaient en tant que dirigeantes d'entreprises. Les résultats de ces travaux, en particulier ceux de Hisrich et Brush (1984) et de Chaganti (1986) ont, dès lors, permis de formuler l'hypothèse de l'existence d'un style de gestion distinctif chez les femmes. Depuis, des résultats d'études empiriques supportent cette conclusion en démontrant comment les dirigeantes d'entreprises, qu'elles soient propriétaires ou non, ont un

1. Traduction de Proulx (1995). 
style de leadership différent de celui des hommes à bien des égards (Helgesen, 1990 ; Buttner, 2001 ; Carter et Cannon, 1992). Se basant sur la théorie du leadership de Bass, certains (Ladouceur, 1993 ; Folker, 1999) iront jusqu'à dire que le style de leadership des femmes est transformationnel alors que celui des hommes est de nature plus transactionnelle. Cela expliquerait, entre autres, le fait que celles-ci, par leur style de gestion, encourageraient grandement le niveau d'engagement et l'implication au travail de leurs troupes.

Cependant, ce sont les résultats des études réalisées sur la perception que les femmes ont de leurs habiletés à diriger, sur leur système de valeurs, sur leurs objectifs et, finalement, sur leurs critères de performance qui apportent davantage de support à la théorie que les femmes ont une manière bien à elles de gérer leurs entreprises et que l'entrepreneuriat féminin représente, somme toute, un univers différent de l'entrepreneuriat masculin. À ce titre, les quelques recherches sur la perception de leurs compétences comme gestionnaire révèlent que les femmes disent posséder d'excellentes compétences reliées aux domaines du leadership et de la gestion des ressources humaines. Elles mentionnent avoir, entre autres, des habiletés en communication, avoir de la facilité pour les relations interpersonnelles et pour le travail d'équipe (Brush et Hisrich, 2000; Collerette et Aubry, 1988 ; Helgesen, 1990; Hisrich et Brush, 1984). Accordant plus d'importance que leurs homologues masculins aux valeurs sociales et humaines, leur structure valorielle expliquerait en partie leur style de gestion, dit féminin. Cela se reflète par les objectifs qu'elles poursuivent et par les critères qu'elles utilisent pour évaluer leur réussite en affaires. En effet, en plus de poursuivre des objectifs économiques, les femmes entrepreneures ciblent des objectifs non économiques se révélant aussi importants, sinon plus que les premiers. Ces objectifs sont la qualité de vie, la qualité des relations interpersonnelles qu'elles entretiennent, en particulier avec leurs employés et leurs clients, et leur développement personnel (Brush, 1992; Lee-Gosselin et Grisé, 1990). Autrement dit, les femmes se donnent un amalgame d'objectifs économiques, personnels et sociaux, les différenciant ainsi grandement des hommes. Pour elles, la réussite en affaires ne se calcule pas uniquement par des indicateurs classiques comme le profit, le chiffre d'affaires, le nombre d'employés et le retour sur investissement, mais aussi par la contribution sociale, la qualité de vie au travail et la satisfaction de la clientèle. Pour les tenants de l'approche intégrée, les femmes ont donc un mode de fonctionnement leur appartenant, tant dans leur manière d'interagir avec les autres que dans leur façon de diriger leurs entreprises et les résultats des études dont nous venons de traiter confortent la thèse qu'effectivement il existerait un modèle féminin de gestion en contexte de PME, lequel découle des comportements de gestion dits relationnels, coopératifs et participatifs chez les femmes entrepreneures versus un mode dominateur, compétitif et directif chez les entrepreneurs masculins.

Revue internationale P.M.E., vol. 15, nº 1, 2002 


\section{Les femmes entrepreneures en contexte d'entreprises familiales}

À ce jour, l'ensemble des travaux réalisés sur les femmes entrepreneures ont principalement cherché à apporter des réponses à des questions touchant l'émergence de ce phénomène, en apparence nouveau pour les chercheurs. Leur questionnement touchait, en grande partie, la réalité entrepreneuriale de ces femmes à des moments précis de l'évolution de leurs entreprises, à savoir les stades de démarrage et de croissance alors qu'au même titre que celles dirigées par des hommes, les PME appartenant à des femmes passeront de la phase entrepreneuriale à celle de PME familiales (Gersick et al., 1997; Hugron, 1998), cela principalement par la venue officielle d'un des membres de leurs familles dans l'entreprise, dans le but précis de prendre la relève et d'en assurer la continuité. Or, à la suite d'une recension exhaustive de la littérature, tant sur les entreprises familiales que sur les femmes entrepreneures, il appert que les recherches unissant les deux champs de recherches sont quasi inexistantes (Cadieux, 1999). Dans certains cas, en contexte d'entreprises familiales, sous prétexte d'éviter un biais d'analyse, on ira jusqu'à soustraire de l'échantillon original les entreprises dirigées par des femmes alors qu'elles occupent une place de plus en plus importante dans toutes les sphères de ces mêmes organisations (Friskhoff et Brown, 1993). Voilà pourquoi nous nous permettons de dire que les connaissances acquises sur les entreprises familiales et, plus particulièrement, sur la problématique de la succession, ne sont valables que pour des entreprises principalement dirigées par des hommes. Au même titre que nous nous permettons de stipuler que nous ignorons pratiquement tout de la réalité des entreprises familiales dirigées par des femmes qui, elles aussi, sont à une étape de leur vie où elles réfléchissent au destin de leurs entreprises (Brush et Bird, 1996).

\subsection{Les perspectives de recherche en entreprises familiales}

A priori, la dynamique des entreprises familiales est particulière et complexe, principalement par la présence indissociable de deux principaux sous-systèmes, soit la famille et l'entreprise. Mais, dans la littérature, il existe deux manières de l'aborder. Considérant la famille et l'entreprise comme deux sous-systèmes interreliés et distincts, la première tient pour acquis que les différences existant entre ceux-ci sont des sources de conflit presque inévitables. En conséquence, vu sous cet angle, les membres de la famille impliqués dans l'entreprise sont confrontés à deux manières d'agir selon le sous-système dans lequel ils se retrouvent, provoquant ainsi une constante ambiguïté dans les rôles et les fonctions de chacun (Kaslow et Kaslow, 1992 ; Kets de Vries, 1993 ; Rosenblatt et al., 1985). Bien que cette perspective ait permis l'avancement des connaissances dans le domaine des entreprises familiales, trop utilisée, elle suscite, selon Whiteside et Brown (1991), trois principales erreurs conceptuelles, à savoir le développement d'une perception stéréotypée des deux

Revue internationale P.M.E., vol. 15, nº 1, 2002 
principaux sous-systèmes, une analyse inconsistante et inadéquate des dynamiques interpersonnelles et, finalement, une exagération des notions de frontières existant entre les sous-systèmes.

Pour sa part, la seconde perspective prône une approche plus intégrée dans la mesure où la famille et l'entreprise sont considérées comme deux sous-systèmes évoluant ensemble (Kepner, 1983 ; Whiteside et Brown, 1991). Pour les tenants de cette approche, l'un ne va pas sans l'autre dans la mesure où la famille, son cycle de vie, ses objectifs et ses manières d'être sont naturellement transposés en contexte organisationnel. Pour eux, dissocier les deux sous-systèmes est, à la limite, impossible et étudier la dynamique des entreprises familiales n'est faisable qu'à la condition de considérer les deux principaux sous-systèmes comme un tout, l'un n'allant pas sans l'autre, ce qui, à notre avis, rejoint grandement la philosophie proposée par Brush (1992) quant au modus operandi des femmes en affaires.

\subsection{La succession dans les entreprises familiales}

Par définition, la succession est un processus dynamique durant lequel les rôles et les fonctions des deux principaux groupes d'acteurs, soit le prédécesseur et le successeur $^{2}$, évoluent de manière dépendante et imbriquée, cela dans le but ultime de transférer à la prochaine génération, d'une part, la gestion de l'entreprise et, d'autre part, la propriété. Partant du principe que le processus débute bien avant l'intégration du successeur dans l'entreprise et se termine lors du désengagement définitif du prédécesseur, les phases indiquées dans les modèles existants (Barnes et Hershon, 1976; Longenecker et Schoen, 1978; Churchill et Hatten, 1987 ; Handler, 1990 ; Hugron et Dumas, 1993) peuvent, comme l'illustre la figure 1, être résumées par les quatre suivantes : l'initiation, l'intégration, le règne conjoint et le désengagement.

Durant la première phase, le propriétaire-dirigeant, souvent seul, a comme principale préoccupation la gestion courante et complète de l'entreprise. Même si le successeur n'a pas de place apparente dans l'entreprise familiale, c'est toutefois durant cette période qu'il y est initié et qu'il développe une perception positive de son parent comme dirigeant (Matthews, Moore et Fialko, 1999). Souvent, c'est durant cette phase que le successeur commence à démontrer de l'intérêt pour l'entreprise pour autant que ce dernier fasse un choix implicite de celui qui prendra la relève (Hugron et Dumas, 1993 ; Matthews, Moore et Fialko, 1999). La deuxième phase débute habituellement lorsque le propriétaire-dirigeant intègre le successeur dans l'entreprise dans un poste à temps partiel ou saisonnier. Considéré en période

2. Dans la mesure où les connaissances acquises proviennent d'échantillons principalement composés d'hommes, le masculin a été privilégié dans la présentation du processus de la succession et des sources de résistances. Du reste, le singulier étant utilisé dans le but d'alléger le texte, il peut s'agir de un ou de plusieurs prédécesseurs et successeurs.

Revue internationale P.M.E., vol. 15, $\mathrm{n}^{\mathrm{o}} 1,2002$ 
d'apprentissage, ce dernier est alors souvent confiné à des tâches de subalterne et très peu impliqué dans les décisions opérationnelles (Longenecker et Schoen, 1978; Handler, 1990; Hugron et Dumas, 1993).

La troisième phase, considérée comme l'étape charnière du processus, se différencie des précédentes, principalement par l'entrée officielle du successeur à ce titre dans l'entreprise (Stravou, 1998). Alors, le prédécesseur et le successeur travaillent côte à côte, assurant ainsi le transfert des responsabilités et de l'autorité, et cela jusqu'à ce que le successeur devienne autonome dans les décisions concernant l'entreprise. Enfin, la dernière phase du processus est, en théorie, caractérisée par le retrait officiel du prédécesseur et par le transfert complet des responsabilités (Churchill et Hatten, 1987; Hugron et Dumas 1993), du leadership, de l'autorité (Handler, 1990) et, à la limite, de la propriété (Churchill et Hatten, 1987 ; Hugron et Dumas, 1993).

FIGURE 1

Le processus de la succession

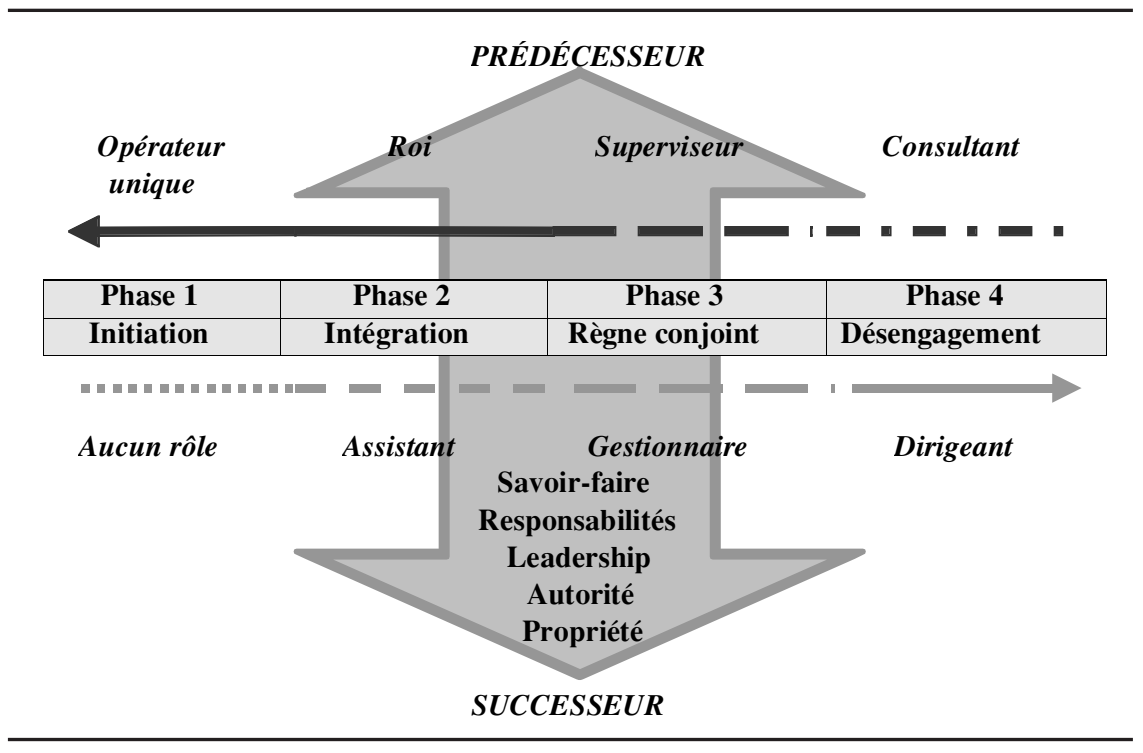

\subsection{Les sources de résistance}

Selon les observations de Handler (1994), il est courant pour le prédécesseur de stopper le processus de la succession à la deuxième ou à la troisième phase. Cela veut dire que, par effet de dépendance des ajustements de rôles proposés par Handler (1990), le successeur n'assumera jamais tout à fait le rôle de dirigeant lui incom- 
bant à la phase du désengagement. De ce fait, certains transferts inhérents à cette même phase ne seraient jamais entièrement complétés tant et aussi longtemps que le prédécesseur serait capable de les assumer (Barnes et Hershon, 1976; Davis et Harveston, 1998; Hugron et Dumas, 1993).

Nonobstant des raisons d'âge ou de santé, pour plusieurs (Barnes et Hershon, 1976 ; Kets de Vries, 1977, 1993 ; Lansberg, 1988 ; Peay et Dyer, 1989; Rubenson et Gupta, 1996), ces sources de résistances sont étroitement liées aux caractéristiques psychologiques si particulières aux entrepreneurs. Par exemple, il est fort probable que l'entrepreneur ayant consacré le plus clair de son temps à son entreprise n'ait jamais eu l'occasion de développer d'autres centres d'intérêts et cela a de l'importance, car plus son éventail est large, plus il se détache facilement de son entreprise. En outre, à cause de certaines craintes non apprivoisées, comme la peur de la mort, de vieillir, de perdre son identité et de perdre ses pouvoirs, le prédécesseur ferait, de manière inconsciente, tout ce qu'il peut pour retarder l'enclenchement du processus. Ainsi, est-il permis de faire un lien entre les caractéristiques psychologiques et comportementales du prédécesseur et le transfert de ses besoins affectifs sur son entreprise et de supposer qu'un des principaux obstacles à la planification ou à la complétude de la succession découle de sa difficulté à lâcher prise.

Toutefois, au tableau 1 , sont indiquées d'autres sources de résistances devant être prises en compte. Par exemple, les résultats de recherches empiriques montrent qu'outre la présence d'un successeur ayant les compétences requises pour prendre la relève (Chrisman, Chua et Sharma, 1998 ; Sharma, Chua et Chrisman, 2000) c'est avant tout la qualité de la relation existant entre les deux principaux intéressés et parmi tous les membres de la famille qui est garante du succès du transfert de l'entreprise à la génération suivante (Davis et Harveston, 1998 ; Dunn, 1999; Lansberg et Astrachan, 1994; Morris et al., 1997). De plus, nous ne pouvons passer sous silence les environnements interne et externe de l'organisation, dans la mesure où des obstacles importants peuvent, entre autres, provenir des employés, du personnel cadre, des clients ou des fournisseurs qui, au fil des ans, ont développé des relations étroites et tissé des liens de confiance avec le prédécesseur (Lansberg, 1988).

\section{Discussion}

Depuis que le monde des affaires existe, les entreprises familiales sont présentes; elles seraient même « les organisations les plus vieilles au monde et les plus répandues » (Hugron, 1998). Présentes dans tous les secteurs d'activité, au Canada seulement elles génèrent $45 \%$ du PNB, procurent de l'emploi à $50 \%$ de la maind'œuvre et créent annuellement entre $70 \%$ et $85 \%$ des nouveaux emplois (CAFE, 2000). Or, qu'elles soient canadiennes, américaines ou européennes, la plupart ayant été fondées après la Seconde Guerre mondiale sont, à l'heure actuelle, confrontées à un problème de taille, celui de la succession (Allouche et Amann, 1998).

Revue internationale P.M.E., vol. 15, nº 1, 2002 
TABLEAU 1

\section{Les sources de résistance}

\begin{tabular}{ll}
\hline Individuelles & $\begin{array}{l}\text { Caractéristiques psychologiques et comportementales du } \\
\text { propriétaire-dirigeant : valeurs, centres d'intérêts, besoins } \\
\text { de contrôle, peurs, degré d'identification à l'entreprise, objectifs } \\
\text { personnels, santé, âge, cycle de vie. }\end{array}$ \\
\hline Interpersonnelles & $\begin{array}{l}\text { Q } \\
\text { et paralité de la relation existant entre le prédécesseur et le successeur } \\
\text { de la communication, le degré de confiance, le respect mutuel, } \\
\text { l'affabilité. }\end{array}$ \\
\hline Organisationnelles & $\begin{array}{l}\text { Culture, structure, liens existants entre le prédécesseur } \\
\text { et le personnel de soutien, le personnel cadre, les membres } \\
\text { du conseil d'administration. }\end{array}$ \\
\hline Environnementales & $\begin{array}{l}\text { Fournisseurs, clients, concurrents gouvernements, institutions } \\
\text { financières, marché, etc. }\end{array}$ \\
\hline
\end{tabular}

La révision de la littérature sur les sources de résistance révèle qu'un des facteurs importants de l'échec du processus de la succession provient de la difficulté de lâcher prise du prédécesseur, principalement à cause de ses craintes et du sentiment d'une perte d'identité que suscite le retrait des affaires. Pour les entrepreneurs, leur estime de soi, leur valorisation personnelle et leur identité reposent en grande partie sur les résultats de leurs activités professionnelles. Souvent, les entrepreneurs n'ont qu'un seul centre d'intérêt dans la vie : leur entreprise. Connaissant l'importance qu'accordent les hommes à leurs intérêts professionnels et à leur carrière, en la délaissant, ceux-ci se retrouvent devant un néant existentiel : en dehors de leur travail, de leur entreprise, plus de vie intéressante (Hansson et al., 1997 ; Levinson et Wofford, 2000). Les femmes, par la place qu'elles occupent dans la société, s'investissent personnellement dans les deux principales sphères les concernant, soit la famille et l'entreprise et, contrairement aux hommes, développent d'autres centres d'intérêts que ceux liés au domaine des affaires. Pour elles, réussir leur vie ne repose pas uniquement sur leur réussite professionnelle, mais aussi sur la réussite de leur vie personnelle et familiale. Finalement, sachant qu'en général les femmes ont plus de facilité à prendre leur retraite (Calasanti, 1996; Feldman, 1994), cet aspect soulève déjà, à lui seul, un questionnement de taille. En effet, qu'en est-il des femmes entrepreneures? Démontrent-elles des difficultés à quitter leur entreprise? Comment vivent-elles cette étape de leur vie sur le plan intrapsychique? Ont-elles ces craintes? Vivent-elles cette perte identitaire?

Par ailleurs, le processus de succession est fondamentalement une question de rapports interpersonnels entre le prédécesseur et le successeur, entre le parent et l'enfant. Les rapports des pères entrepreneurs avec les enfants sont habituellement différents des relations entre les mères entrepreneures et leurs enfants. Pour les 
hommes et les femmes, la réalité est différente. Par exemple, des résultats d'enquêtes révèlent que même si $90 \%$ des femmes propriétaires d'entreprises travaillent à temps complet et que $25 \%$ y consacrent plus de soixante-dix heures par semaine, elles demeurent les premières responsables de la gestion et de l'exécution des tâches ménagères et de l'éducation des enfants (Belcourt, Burke et Lee-Gosselin, 1991). Les mères sont toujours en relation avec leurs enfants alors que les pères sont plutôt absents, trop préoccupés par leurs affaires (Wallott, 1984). Pour elles, jouer simultanément les rôles de dirigeante d'entreprise et d'éducatrice est chose commune. Elles voient leurs enfants grandir, évoluer, se développer. Étant souvent les premières personnes vers qui ils se tournent dans les moments difficiles de leur existence, elles vivent avec eux leurs réussites et leurs échecs, leurs joies et leurs peines. Somme toute, elles connaissent bien qui et comment sont leurs enfants : leur personnalité, leurs intérêts, leurs ambitions et leurs potentiels. Cela laisse donc entrevoir la possibilité de l'existence d'une dynamique différente en contexte de succession dans la mesure où, dans le cas des entreprises dirigées par des femmes, la relation entre les deux principaux intéressés est déjà bien établie, contrairement à celle existant entre les pères et leurs enfants lesquels, en définitive, apprennent souvent à se connaître réellement lorsqu'ils doivent travailler ensemble.

Dans la littérature, une des raisons pour lesquelles les prédécesseurs retarderaient l'enclenchement du processus de la succession découle d'un souci d'équité pour chacun des enfants et d'une crainte de l'apparition de conflits familiaux (Friedman, 1991). Bien sûr, la succession a des incidences sur la dynamique familiale. Il n'est pas rare de voir la succession provoquer la désintégration du tissu familial ou la rupture de la cellule familiale. Devant cette menace, comment réagissent les femmes entrepreneures ? Elles qui ont toujours vu à l'unité, à la cohésion familiale et à l'harmonie des relations entre tous les membres de la famille (Lacourse, 1994). Sauront-elles se prémunir contre ce danger? Comment? Est-ce que leurs compétences sociales leur permettront de gérer efficacement, le cas échéant, la crise familiale engendrée par la succession? Pour avoir été celles ayant établi les liens existants entre tous les membres de la famille (Lacourse, 1994), les femmes entrepreneures ont aussi une excellente compréhension de la dynamique familiale. Comment cette connaissance des enfants et de la dynamique familiale intervient-elle dans le processus successoral ? Comment les femmes entrepreneures gèrent-elles les relations entre le successeur et les autres membres de la fratrie ? Comment les femmes gèrent-elles l'interface famille-entreprise?

En contexte d'entreprises familiales, il appert que les hommes ont une forte tendance à préférer leur garçon au détriment de leur fille, et ce, indépendamment de leurs compétences et de leurs intérêts (Dumas, 1990; Seymour, 1993). La décision du choix du successeur est un processus fondé sur des critères subjectifs et inconscients, dont celui provenant d'un biais masculin. Mais lorsque cette décision est prise par des femmes, celles-ci, semblables à leurs homologues masculins,

Revue internationale P.M.E., vol. 15, n ${ }^{\circ} 1,2002$ 
sont-elles influencées par ce biais masculin dans le choix de leurs successeurs ? Ou au contraire, ayant souvent dénoncé avoir été victimes de discrimination de la part des hommes dans le monde des affaires, sont-elles plus influencées par un biais féminin, de façon à affirmer la position des femmes dans le monde de l'entrepreneuriat?

La succession a également des effets sur la dynamique organisationnelle et des répercussions sur le système social de l'entreprise. Par exemple, l'intégration des enfants dans l'entreprise familiale dans le but spécifique de les préparer à prendre la relève peut certes déplaire à certains employés loyaux et en place depuis plusieurs années. Prenant conscience de la fin de leurs ambitions de carrière ou de leurs possibilités d'avancement, ceux-ci adoptent souvent, par frustration, des comportements nuisibles au bon déroulement du processus. Les femmes entrepreneures ayant, par nature, un style de gestion participatif et transformationnel tissent, au fil des ans, des liens serrés avec plusieurs de leurs employés. Ayant toujours à cœur le bien-être et l'épanouissement de ceux-ci, ayant toujours manifesté de la reconnaissance pour leur contribution à l'essor de l'entreprise, elles vivent, sans doute, un conflit intérieur les obligeant à choisir entre leurs employés et leurs enfants. Comment gèrent-elles les choix qui se posent à elles ? Comment gèrent-elles les résistances provenant de ces employés jadis loyaux ? En outre, à l'exception de cette source de résistance potentielle, nous devons nous questionner sur la manière dont elles impliquent ce même personnel dans le processus de socialisation et d'intégration de leurs enfants dans l'entreprise. Sont-elles sensibles aux activités de parrainage ? Apparient-elles des employés à leurs enfants pour que ces derniers puissent faire les apprentissages requis ou, au contraire, s'approprient-elles la formation de leurs enfants, leurs successeurs?

\section{Conclusion}

Aborder la problématique de la succession dans les entreprises familiales sous l'angle de l'entrepreneuriat féminin remet en question l'état de nos connaissances et suscite un questionnement de taille. Faute de recherches empiriques, la communauté scientifique doit s'avouer ignorante à ce chapitre alors que cette réalité nous interpelle. Sachant qu'il y a, dans un avenir rapproché, un nombre considérable de femmes qui vivront avec acuité les difficultés de la succession (Frishkoff et Brown, 1993 ; Brush et Bird, 1996), nous devons nous interroger sur la capacité des spécialistes en entrepreneuriat et en gestion des PME d'intervenir de manière efficace et professionnelle dans cette sphère.

La parcimonie des recherches sur l'entrepreneuriat féminin provoque également une prise de conscience de nos limites et de nos partis pris en tant que chercheurs dans le domaine et, en particulier, ceux de la communauté francophone. Comment pouvons-nous expliquer notre désintéressement à l'égard de ce phénomène qu'est l'entrepreneuriat féminin ? Comment expliquer que, pour la plupart, l'entrepreneuriat,

Revue internationale P.M.E., vol. 15, $\mathrm{n}^{\circ} 1,2002$ 
au même titre que l'entreprise familiale, se conjugue seulement au masculin ? Depuis plusieurs décennies, les rapports officiels de nos gouvernements et de certaines agences nationales et internationales montrent, sans l'ombre d'un doute, l'ampleur de ce phénomène et, de plus, prévoient que d'ici quelques décennies, une entreprise sur deux sera dirigée par une femme. Comment pouvons-nous expliquer cet écart entre notre niveau de connaissances sur ce sujet et l'importance de ce phénomène?

Entreprendre des recherches sur la succession dans les entreprises familiales dirigées par des femmes remet aussi en question nos pratiques de recherche. Effectivement, la stratégie de recherche la plus courante en contexte d'entrepreneuriat et d'entreprises familiales est celle d'enquête par sondage ex post facto utilisant des échantillons de convenance. À notre avis, pour comprendre et aborder les particularités intrinsèques de ce phénomène, des stratégies de recherche d'ordre plus qualitatif sont indispensables, voire incontournables. Pour comprendre la réalité des femmes entrepreneures confrontées à la succession de leurs entreprises, il faut faire des études de cas en profondeur et les outils nécessaires pour mener à bien de telles recherches sont à notre portée. Par exemple, alors que Yin (1994) et Eisenhardt (1989) proposent une démarche empirique intéressante pour l'étude d'un phénomène contemporain, évolutif et complexe comme celui qui nous intéresse, Langley (1999) indique, pour sa part, plusieurs méthodes pouvant aider les chercheurs à analyser rigoureusement de tels types de recherches dites processuelles.

La problématique de la succession dans les entreprises familiales dirigées par des femmes est présente, réelle. Nous avons les outils nécessaires pour mener à bien ces recherches. Il ne reste qu'à trouver des chercheurs y croyant et voulant s'investir dans l'avancement des connaissances.

\section{Bibliographie}

Allouche, J. et B. AmANN (1998), «La confiance : une explication des performances des entreprises familiales », Économie et société, vol. 8, nº 9, p. 129-154.

BAKER, T., H.-E. ALDRICH et N. LIOU (1997), «Invisible entrepreneurs : the neglect of women owners by mass media and scolarity journals in USA », Entrepreneurship Theory and Practice, vol. 9, $\mathrm{n}^{\mathrm{o}} 3$, p. 221-238.

BARNES, L.-B. et S.-A. HERSHON (1976), « Transferring power in family business », Harvard Business Review, p. 105-114.

Belcourt, M., R. Burke, et H. LeE-Gosselin (1991), Une cage de verre : les entrepreneures au Canada, Conseil consultatif canadien sur la situation de la femme.

BRUSH, C. (1992), «Research on women business owners : past trends, and new perspective and future directions », Entrepreneurship Theory and Practice, vol. 16, no 4, p. 5-30.

BRUSH, C. et B.-J. BIRD (1996), «Leadership vision of successful women entrepreneurs : dimensions and characteristics », Frontiers of Entrepreneurship Research, http :// www.babson.edu/entrepre/fer/papers96/summ96/brush.html.

Revue internationale P.M.E., vol. 15, nº 1, 2002

(C) 2002 - Presses de l'Université du Québec

Édifice Le Delta I, 2875, boul. Laurier, bureau 450, Sainte-Foy, Québec G1V 2M2 • Tél. : (418) 657-4399 - www.puq.uquebec.ca

Tiré de : Revue internationale P.M.E., vol. $15, n^{\circ} 1$, sous la direction de Pierre-André Julien. 
BRUSH, C.-G. et R.-D. HISRICH (2000), «Women-owned businesses : an exploratory study comparing factors affecting performance », RISEbusiness, Research Institute for Small \& Emerging Business Inc., Working Papers Series 00-02, www.riseb.org.

BUTTNER, E.-H. (2001), «Examining female entrepreneurs' management style : an application of a relational framework », Journal of Business Ethics, vol. 29, p. 253-269.

CADIEUX, L. (1999), La succession en entreprise familiale : Analyse du processus dans le cas de quatre entreprises manufacturières fondées par des femmes, Université du Québec à Trois-Rivières.

CAFE (2000), « History and mandate : profile/overview », Canadian Association of Family Enterprise, http ://www.cafenational.org/nat_history.html.

Calasanti, T.-M. (1996). "Gender and life satisfaction in retirement: an assessment of the male model », Journal of Gerontology, vol. 51B, n 1, p. S28-S29.

CARTER, S. et N.T. CANNON (1992), Women as Entrepreneurs, Londres, Academic Press, Harcourt Brace Jovanovich Publishers.

Chaganti, R. (1986), «Management in women-owned enterprises », Journal of Small Business Management, vol. 24, no 4, p. 19-29.

Chrisman, J.-J., J.-H. CHUA, et P. SHARMA (1998), «Important attributes of successors in family businesses : an exploratory study », Family Business Review, vol. XI, n 1 , p. 19-34.

Churchill, N.-C. et K.-J. HATTEN (1987), « Non-market based transfers of wealth and power : a research framework for family businesses », American Journal of Small Business, vol. 11, $\mathrm{n}^{\mathrm{0}} 3$, p. 51-64.

Collerette, P. et P. Aubry (1988), Femmes et hommes d'affaires, qui êtes-vous?, Montréal, Agence d'Arc.

DAVIS, P.-S. et P.-D. HARVESTON (1998), « The influence of family on the family business succession process : a multi-generational perspective », Entrepreneurship Theory and Practice, vol. 22, $\mathrm{n}^{\circ}$ 3, p. 31-53.

DuMAS, C. (1990), « Preparing the new CEO : managing the father-daughter succession process in family businesses », Family Business Review, vol. III, n 2, p. 169-181.

DunN, B. (1999), «The family factor: the impact of family relationship dynamics on business-owning families during transitions », Family Business Review, vol. XII, $\mathrm{n}^{\mathrm{o}} 1$, p. 41-55.

EISENHARDT, K.-M. (1989). «Building theories from case study research », Academy of Management Review, vol. 14, $\mathrm{n}^{\mathrm{o}}$ 4, p. 532-550.

FELdmAN, D.-C. (1994). «The decision to retire early : a review and conceptualization », Academy of Management Review, vol. 19, n 2, p. 285-311.

FOLKER, C.-A. (1999), «Female vs male family business owners : exploring the differences through a trust/distrust framework», USASBE Proceedings, www.sbaer.uca.edu/ docs/proceedingsIII.

FRIEDMAN, S.-D. (1991), « Sibling relationships and intergenerational succession in family firms », Family Business Review, vol. IV, no 1, p. 3-20.

FrishKofF, P.A. et B.-M. BROWN (1993), «Women on the move in family business », Business Horizons, vol. 36, $\mathrm{n}^{\circ}$ 2, p. 66-70.

Revue internationale P.M.E., vol. 15, nº 1, 2002 
Gersick, K.-E., J.-A. Davis, M. McCollom Hampton et I. LANSBerg (1997), Generation to Generation: Life Cycles of the Family Business, Boston, Harvard Business School Press.

HANDLER, W.-C. (1990), «Succession in family firms : a mutual role adjustment between entrepreneur and next-generation family members », Entrepreneurship Theory and Practice, vol. 15, $\mathrm{n}^{\mathrm{o}}$ 1, p. 37-51.

HANDLER, W.-C. (1994), «Succession in family business : a review of the research », Family Business Review, vol. VII, no 2, p. 133-157.

Hansson, R.-O., P.-D. DeKoekKoeK, W.-M. Neece et D.-W. Patterson (1997). «Successful aging at work: annual review 1992-1996: the older worker and transitions to retirement », Journal of Vocational Behavior, vol. 51, n 2, p. 202-233.

Helgesen, S. (1990), The Female Advantage : Women's Ways of Leadership, New York, Doubleday.

HISRICH, R.D. et C. BRUSH (1984), «The woman entrepreneur : management skills and business problems », Journal of Small Business Management, vol. 22, no 1, p. 30-37.

Hugron, P. (1998), «La régie d'entreprises familiales », Gestion, vol. 23, n 3, p. 37-40.

Hugron, P. et C. Dumas (1993), «Modélisation du processus de succession des entreprises familiales québécoises », Groupe de recherche sur les entreprises familiales, Cahier de recherche ${ }^{\circ}$ GREF-93-07, Hautes Études commerciales.

KASLOW, F.-W. et S. KASLOW (1992), « The family that works together : special problems of family business », dans Work, Families and Organizations, San Francisco, JosseyBass, p. 312-361.

KEPNER, E. (1983), «The family and the firm : a coevolutionary perspective », Organizational Dynamics, vol. 12, p. 57-70.

KETS DE VRIES, M.-F. (1977), «The entrepreneurial personality : a person at the crossroads », The Journal of Management Studies, vol. 14, p. 34-57.

KETS DE VRIES, M.-F. (1993), «The dynamics of family controlled firms: the good and the bad news », Organizational Dynamics, p. 59-71.

LACOURSE, M.-T. (1994), Famille et Société, Montréal, Chenelière/McGraw-Hill.

LADOUCEUR, N. (1993), «Hommes ou femmes : qui est le meilleur gestionnaire », dans MICST (2000), L'entrepreneuriat féminin : une force un atout. Portrait statistique des femmes entrepreneures, Québec, Ministère de l'Industrie et du Commerce.

LANGLEY, A. (1999), «Strategies for theorizing from process data », Academy of Management Review, vol. 24, n 4, p. 691-710.

LANSBERG, I. (1988), «The succession conspiracy », dans Family Business Sourcebook II, (1996). Publié par Aronoff, Astrachan et Ward, Marietta, Georgia, Business Owner Resources, p. 70-86.

LANSBERG, I. et J.-H. ASTRACHAN (1994), «Influence of family relationships on succession planning and training: the importance of mediating factors », Family Business Review, vol. VII, no 1, p. 39-59.

LEE-Gosselin, H. et J. GRISÉ (1990), « Are women owner-managers challenging our definition of entrepreneurship? An in-depth study », Journal of Business Ethics, vol. $9, \mathrm{n}^{\text {os }} 4-5$, p. 423-433.

Revue internationale P.M.E., vol. 15, n 1, 2002 
LEVINSON, H. et J.-C. WOFFORD (2000), « Approaching retirement as the flexibilty phase », The Academy of Management Executive, vol. 14, n 2, p. 84-95.

Longenecker, J.-G. et J.-E. SCHOEN (1978), «Management succession in the family business », dans Family Business Sourcebook II (1996). Publié par Aronoff, Astrachan et Ward, Marietta, Georgia, Business Owner Resources, p. 87-92.

Matthews, C.-H., T.-W. Moore et A.-S. FialKo (1999), « Succession in the family firm : a cognitive categorization perspective », Family Business Review, vol. XII, nº 2, p. $159-169$.

MINISTÈRE DE L'INDUSTRIE ET DU COMMERCE (2000), L'entrepreneuriat féminin : une force un atout. Portrait statistique des femmes entrepreneures, Québec, Ministère de l'Industrie et du Commerce.

Morris, M.-H., R.-O. Williams, J.-A. Allen et R.-A. Avila (1997), «Correlates of success in family business in transition », Journal of Business Venturing, vol. 12, no 5 , p. 385-401.

PEAY, R.-T. et G. DYER (1989), «Power orientations of entrepreneurs and succession planning », Journal of Small Business Management, vol. 27, $\mathrm{n}^{\circ}$ 1, p. 47-52.

PROUlX, S. (1995), La problématique de l'entrepreneurship féminin : quelques éléments de base. Recension et rapport-synthèse des écrits, Québec, La corporation Jonathan 1445.

RATTÉ, S. (1999), «Les femmes entrepreneures au Québec : qu'en est-il ? , Fédération canadienne de l'entreprise indépendante, Montréal, dans MICST (2000), L'entrepreneuriat féminin: une force un atout: portrait statistique des femmes entrepreneures, Québec, Ministère de l'Industrie et du Commerce.

Rosenblatt, P.C., L. DeMik, R.-M. ANDERSOn et P.-A. Johnson (1985), The Family in Business: Understanding and Dealing with the Challenges Entrepreneurial Families Face, San Francisco, Jossey-Bass.

Rubenson, G.-C. et A.-C. GuPTA (1996), «The initial succession : a contingency model of founder tenure », Entrepreneurship Theory and Practice, vol. 21, n ${ }^{\circ}$ 2, p. 21-35.

SEYMOUR, K.-C. (1993). «Intergenerational relationships in the family firm : the effect of leadership succession », Family Business Review, vol. VI, n 3, p. 263-281.

Sharma, P., J. ChUA et J. Chrisman (2000), « Perceptions about the extent of succession planning in Canadian family firms », Revue canadienne des sciences de l'administration, vol. 17, $\mathrm{n}^{\mathrm{o}} 3$, p. 233-243.

STEVENSON, L. (1990), «Some methodological problems associated with researching women entrepreneurs », Journal of Business Ethics, vol. 9, n 4-5, p. 439-446.

StRavou, E.-T. (1998), «A four-factor model: a guide to planning next generation involvement in the family firm », Family Business Review, vol. XI, n² 2, p. 135-141.

YIN, R.-K. (1994), Case Study Research : Design and Method, $2^{2}$ édition, Thousand Oaks, Californie, Sage.

WALlOTt, H. (1984). «Les aspects humains de la crise dans la petite entreprise », Revue $P M O$, vol. 1, no 2, p. 12-17.

Whiteside, M.-F. et F.-H. BROWN (1991), «Drawbacks of a dual systems approach to family firms : can we expand our thinking?», Family Business Review, vol. IV, no 4 , p. 383-395.

Revue internationale P.M.E., vol. 15, $\mathrm{n}^{\circ}$ 1, 2002 